\title{
Proteomic changes and endophytic micromycota during storage of organically and conventionally grown carrots
}

Louarn, Sébastien Jean Yves; Nawrocki, Arkadiusz; Thorup-Kristensen, Kristian; Lund, Ole Søgaard; Jensen, Ole N.; Collinge, David B.; Jensen, Birgit

Published in:

Postharvest Biology and Technology

DOI:

10.1016/j.postharvbio.2012.08.011

Publication date:

2013

Document version

Early version, also known as pre-print

Citation for published version (APA):

Louarn, S. J. Y., Nawrocki, A., Thorup-Kristensen, K., Lund, O. S., Jensen, O. N., Collinge, D. B., \& Jensen, B.

(2013). Proteomic changes and endophytic micromycota during storage of organically and conventionally grown carrots. Postharvest Biology and Technology, 76, 26-33. https://doi.org/10.1016/j.postharvbio.2012.08.011 


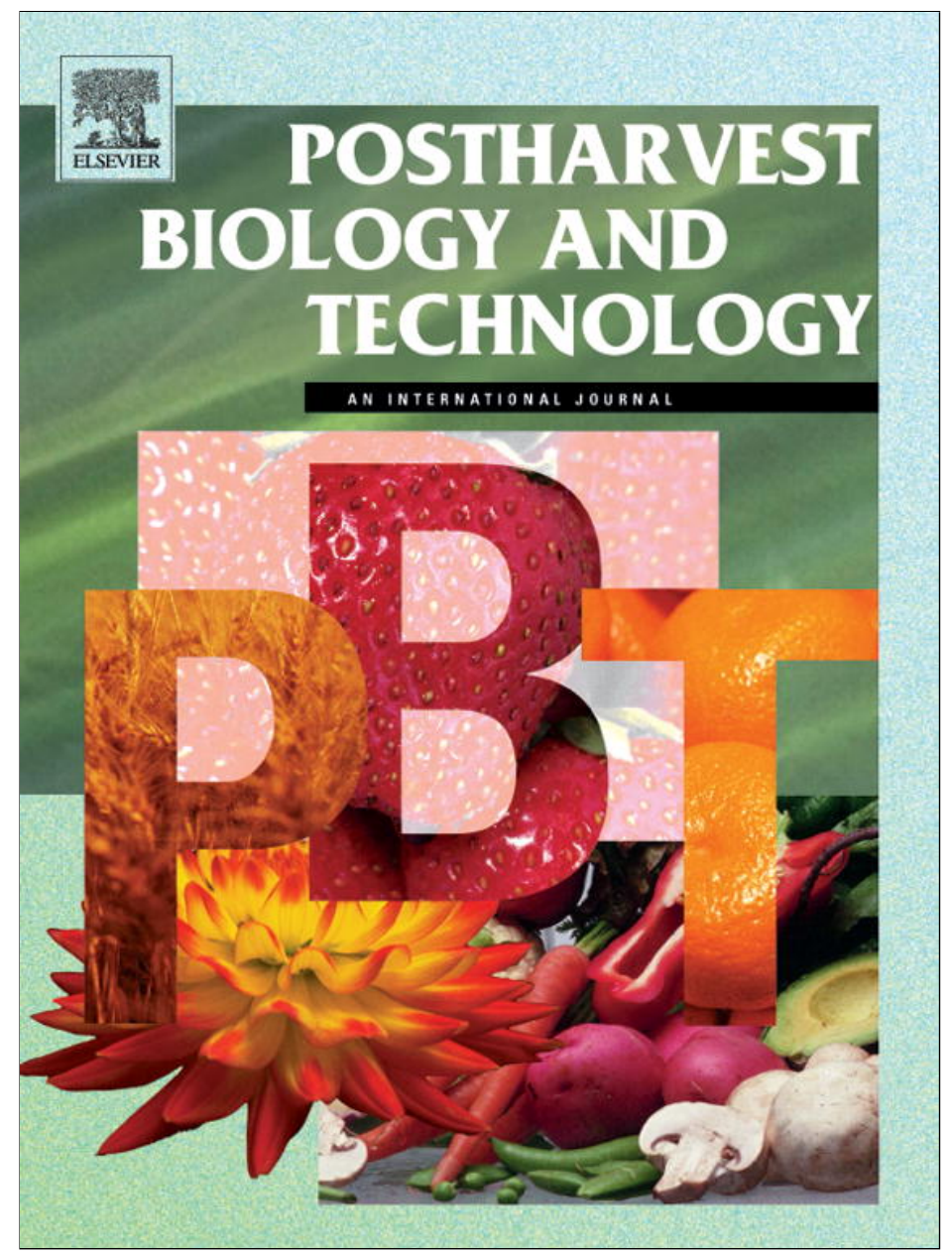

(This is a sample cover image for this issue. The actual cover is not yet available at this time.)

This article appeared in a journal published by Elsevier. The attached copy is furnished to the author for internal non-commercial research and education use, including for instruction at the authors institution and sharing with colleagues.

Other uses, including reproduction and distribution, or selling or licensing copies, or posting to personal, institutional or third party websites are prohibited.

In most cases authors are permitted to post their version of the article (e.g. in Word or Tex form) to their personal website or institutional repository. Authors requiring further information regarding Elsevier's archiving and manuscript policies are encouraged to visit: 


\title{
Proteomic changes and endophytic micromycota during storage of organically and conventionally grown carrots
}

\author{
Sébastien Louarn ${ }^{\mathrm{a}}$, Arkadiusz Nawrocki $^{\mathrm{b}}$, Kristian Thorup-Kristensen ${ }^{\mathrm{c}, 1}$, Ole S. Lund ${ }^{\mathrm{d}, 1}$, \\ Ole N. Jensen $^{\mathrm{b}}$, David B. Collinge $\mathrm{a}^{\mathrm{a}, * 1}$, Birgit Jensen ${ }^{\mathrm{a}, 1}$ \\ a Department of Plant Biology and Biotechnology, Faculty of Life Science, University of Copenhagen, 1871 Frederiksberg, Denmark \\ b Department of Biochemistry and Molecular Biology, University of Southern Denmark, Campusvej 55, 5230 Odense M, Denmark \\ ' Department of Horticulture, Aarhus University, Research Centre Aarslev, Kirstinebjergvej 10, Postbox 102, 5792 Aarslev, Denmark \\ ${ }^{d}$ Department of Agriculture and Ecology, Faculty of Life Science, University of Copenhagen, 1871 Frederiksberg, Denmark
}

\section{A R T I C L E I N F O}

\section{Article history:}

Received 7 February 2012

Accepted 26 August 2012

\section{Keywords:}

Carrot proteome

Endophytic micromycota

Organic production

Cold storage

\begin{abstract}
A B S T R A C T
The physiological state of carrot roots during extended cold-storage is decisive for high postharvest quality. We have investigated differences in the proteome and micromycota of organically and conventionally grown carrots during six months of storage. The levels of only 15 proteins changed in level during storage. Proteins involved in cold stress adaptation and cytoskeleton components changed; these changes in specific protein levels occurred mainly during the first month demonstrating adaptation to storage conditions and that the carrots were subsequently stable, indicating stable carrot quality. The changes observed were similar in the two cropping systems.

Using both biological isolation and a fungal PCR targeting the ITS region, we identified several endophytic species belonging to the Ascomycota. The most frequently encountered taxa were Tetracladium, Leptodontidium, Nectriaceae and Phoma which are known to occur as root endophytes or as root-associated fungi. As for the proteomics data, no consistent statistically significant differences in micromycota were observed between the two cropping systems. We conclude that cropping system did not have an influence on the postharvest quality of the carrots during six months of cold storage.
\end{abstract}

(c) 2012 Elsevier B.V. All rights reserved.

\section{Introduction}

The year-round availability of quality carrots is important for the consumer market; therefore continuing technical improvements to extend cold storage are an important developmental challenge for the carrot industry. However, during cold storage, carrot roots become stressed due to the environmental conditions (low temperature and drought); these stress conditions induce modification of the metabolism of the roots and favour the development of certain diseases (Lecam et al., 1993; Kastelein et al., 2007; Louarn et al., 2012). At the same time, there is an increasing interest among consumers for food safety, especially with respect to pesticide residues and potential human pathogens. Furthermore, there is a general increased concern about the state of the environment, especially in relation to environmental impact caused by the excessive use of inorganic fertilisers. Consumers are therefore turning increasingly to organic products as their production does not entail the

\footnotetext{
* Corresponding author.

E-mail address: dbc@life.ku.dk (D.B. Collinge).

1 Present address: Department of Plant and Environmental Sciences, Faculty of Science, University of Copenhagen, Thorvaldsensvej 40, 1871 Frederiksberg, Denmark.
}

use either of pesticides or mineral fertilisers (Woteki et al., 2001; Magnusson et al., 2003).

Relatively little is known about the physiological changes which occur during storage of root vegetables. Those demonstrated include changes in the occurrence of secondary metabolites, for instance, the isocoumarin, 3-methyl-6-methoxy-8-hydroxy3,4-dihydroisocoumarin (6-MM) a phytoalexin (antimicrobial metabolite), which accumulates in carrots following pathogen infection and in response to treatment with various abiotic elicitors (Mercier et al., 1994; Jayaraj et al., 2009). Interestingly, the content of 6-MM from 1 month old stored carrots was significantly higher than after 4 and 6 months of storage (Louarn et al., 2012). Physiological changes due to cold stress (includes chilling $\left(<20^{\circ} \mathrm{C}\right)$ and freezing $\left(<0^{\circ} \mathrm{C}\right)$ temperatures $)$ can also be observed at the protein level (Degand et al., 2009; Neilson et al., 2010). Several of these proteins e.g., dehydrin, heat-shock proteins (HSP) and antifreeze proteins, are known to be involved in abiotic stress responses and have also been observed to accumulate following cold stress (Miernyk, 1997; Kawamura and Uemura, 2003; Griffith and Yaish, 2004; Renaut et al., 2004; Griffith et al., 2005).

Plant roots host a great variety of fungi; roots are not only susceptible to certain soil-borne pathogens, but can also be colonized by symbiotic mycorrhyzae and endophytic micromycota. 
The spectrum of endophytic organisms present in a particular sample of roots will reflect the physiological state of the root and we assume that, if roots differ substantially in their physiological state, they will probably differ in their endophytic micromycota too. In carrot roots, several plant pathogen interactions have been studied (Kora et al., 2005; Kastelein et al., 2007; Louarn et al., 2012) as well as carrot mycorrhyzal interactions (Dumas-Gaudot et al., 2004). Furthermore, Surette et al. (2003) investigated bacterial endophytes in carrots. However, no studies have been conducted on the endophytic micromycota of carrots roots. Endophytic fungi are asymptomatic for at least part of their life cycle but some can be latent pathogens or dormant saprophytes (that change lifestyle during host senescence, Promputtha et al., 2007; Yuan et al., 2010). The majority of endophytic populations are Ascomycetes (Gazis and Chaverri, 2010; Botella and Diez, 2011) as well as the major carrot root pathogens (Farrar et al., 2004; Kastelein et al., 2007; Louarn et al., 2012). Potential benefits of host-endophyte associations include increased resistance to herbivores, pathogens and abiotic stress (Kuldau and Bacon, 2008; Porras-Alfaro and Bayman, 2011) and they can even, in some cases, be used as biological control agents. The root micromycota from plants grown in cold environments have been studied, thus the establishment of plant-fungal association in relatively extreme environments might help to understand the origins and the role of endophytes (Higgins et al., 2007; Rosa et al., 2010). Some endophytes, such as Curvularia protuberata in rice (Redman et al., 2011), are able to increase plant tolerance to cold stress. On the other hand, factors like soil $\mathrm{pH}$, soil humidity, location and crop rotation (D'Amico et al., 2008), the use of fertilisers (Kernaghan et al., 2003) and predictably, the use of fungicides (Sieber and Grünig, 2006) can influence the diversity of the fungal endophytes communities, and especially nitrogen fertilisation might be one of the mechanisms by which agricultural management affects endophyte communities.

We have previously studied the response of organically and conventionally grown carrots at harvest and in response to pathogen attack during subsequent storage (Nawrocki et al., 2011; Louarn et al., 2012). Changes in specific defence-related metabolites including the accumulation of a phytoalexin (6MM) and proteins (PR-protein) were correlated to fungal infection. In contrast, the present study focuses on the differences between organic and conventional cropping systems and utilises physiologically independent assays to provide an objective evaluation as to whether there are indeed substantial differences in the way that organically and conventionally grown carrots develop during storage. Thus quantitative proteomics and sampling the endophytic Ascomycota of stored carrots provide two different assessments of the physiological state and, for the former, dynamics of the physiology of stored carrots. This study thus represents the first study of the endophytic Ascomycota in carrot roots. We show that, though there are some differences, the organically and conventionally cultivated carrots are substantially similar in quality, and that in essence the alterations in quality change concomitantly during storage.

\section{Materials and methods}

\subsection{Plant material}

The rotation experiment was started in the autumn of 2005 on a field grown organically since 1996 , at Årslev, Denmark $\left(10^{\circ} 27^{\prime} \mathrm{E}\right.$ $\left.55^{\circ} 18^{\prime} \mathrm{N}\right)$. The crops used in these rotations are representative of vegetables produced commercially in Denmark. The two cropping systems are described in detail in our previous study (Louarn et al., 2012; Thorup-Kristensen et al., 2012), and comprise a conventional system where synthetic fertilizers, pesticides, herbicides and fungicides were used according to normal practice and an organic system. The roots were harvested in October 2007 and 2008, samples were taken on harvest day and the carrots then placed in cold storage at $1{ }^{\circ} \mathrm{C}$ and $>98 \% \mathrm{RH}$ and roots were sampled one, four and six months after harvest. Sampling was achieved by cutting two $15 \mathrm{~mm}$ cylinders per carrot with a cork borer; this cylinder was cut longitudinally in two equal pieces, one was kept for proteomic and micromycota analysis and the other for secondary metabolite analysis (Louarn et al., 2012). Only the upper $10 \mathrm{~mm}$ of this cylinder was used for analysis. Each sample consisted of 12 parts from six carrots giving approximately $12 \mathrm{~g}$ of fresh tissue per sample for each three biological replicates. The samples were frozen directly in liquid nitrogen and then freeze-dried.

\section{2. $2 \mathrm{D}$ gel electrophoresis and image analysis}

For the proteomic analysis, freeze-dried root samples were used from the 2008 harvest. Gel electrophoresis and image analysis were performed as described by Nawrocki et al. (2001, 2011) with some modifications according to Louarn et al. (2012). Where necessary, manual corrections of the protein spot detection and matching across the gels were made. Thus a 1.5 -fold up or down regulation $(p<0.05)$ of the protein spot volume is taken as the cut-off for further analysis, since a lower level of change is inferred to approach the technical limitations of the technique. The significance of the differences between averaged spot volumes between pair-wise comparisons in organic samples compared to conventional samples at each time point was tested using Student's $t$-test, with $p<0.05$. The same comparison and test was made between the samples at harvest time compared to samples stored 1 month, 4 months and 6 months for each cultivation systems. These two comparisons enable a comparison of the effect of cultivation system on the proteome changes and, independently, the effect of storage time on the proteome changes.

Protein spots whose intensity differed significantly were excised from the gels and digested with trypsin, and the proteins were identified using a 4800 Plus MALDI TOF-TOF Analyser (Applied Biosystems, MDS SCIEX) as in Louarn et al. (2012).

\subsection{Micromycota}

\subsubsection{PCR identification}

Freeze-dried samples of roots stored for one month, from the conventional and organic cropping system harvested in 2007 and 2008, were included. Roots cold-stored for one month were sampled in triplicate from the organic and conventional cropping systems both in 2007 and 2008. For DNA extraction, samples of freeze-dried carrot material were ground in liquid nitrogen and $20 \mathrm{mg}$ was used for DNA isolated using the Nucleon Phytopure kit (Amersham Biosciences, Buckinghamshire, UK) following the manufacturer's instructions, except that $15 \mathrm{mg}$ polyvinylpolypyrrolidone (Sigma) was added to the lysis buffer. The total reaction volume of the PCR reaction was $20 \mu \mathrm{L}$ and contained $1 \times$ Taq polymerase buffer including $1.5 \mathrm{mM} \mathrm{MgCl}_{2}, 0.2 \mathrm{mM}$ of each dNTP, $0.5 \mu \mathrm{M}$ each of the primers FITS-Fw1: GCCTGTTCGAGCGTCATT and FITS-R1: TCCTACCTGATYCGAGGTCAA (Fig. 1), 2 U Taq DNA polymerase (Amplicon, Copenhagen, DK) and 40-80 ng root DNA. The PCR was performed using a Mastercycler personal (Eppendorf). An initial denaturation at $95^{\circ} \mathrm{C}$ for $3 \mathrm{~min}$ was followed by 36 cycles of denaturation at $94^{\circ} \mathrm{C}$ for $20 \mathrm{~s}$, annealing at $63^{\circ} \mathrm{C}$ for $20 \mathrm{~s}$, and extension for $20 \mathrm{~s}$. The PCR was completed with a final extension step at $72{ }^{\circ} \mathrm{C}$ for $7 \mathrm{~min}$. The PCR products were visualised on a $1.5 \%$ agarose gel. Following the manufacturer's instructions, the PCR products were cloned into the pGEM T-easy vector (Promega, Madison, USA) and transformed into DH5 $\alpha$ competent Escherichia coli cells (Invitrogen, Madison, USA). From each root sample in 2007 and 2008, respectively, 30 colonies and 25 


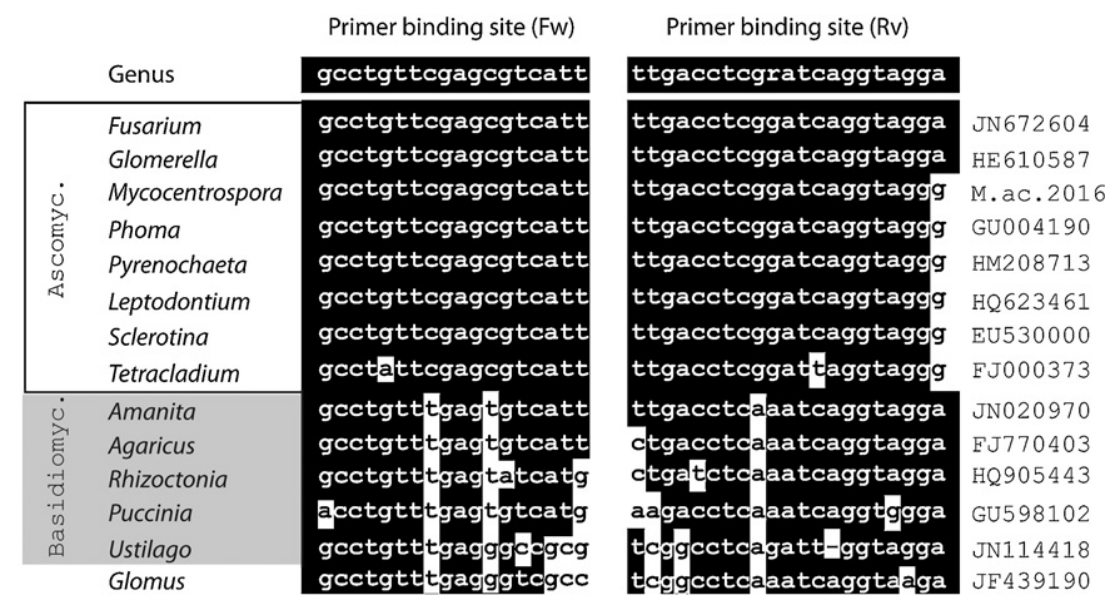

Fig. 1. Alignment of the FITS primers with sequences of Ascomycetes, Basidomycetes and Glomeromycete (mismatches bases in white).

colonies were picked. Colony PCR with the vector primers T7 and Sp6 was performed as described by Sundelin et al. (2009). The PCR products were separated on an agarose gel and clones with band size of approximately $200 \mathrm{bp}$ were sequenced using the sequencing service of StarSeq, Mainz, Germany. Sequences were queried against GenBank and identified in a BLAST search by the National Centre for Biotechnology Information (NCBI). Each sequence was identified to the lowest taxonomic rank common to the top blast hits. Only clone sequences represented at least twice in 2007 and/or 2008 were considered as identified taxa; those occurring only once were assigned to the phylum Ascomycota.

\subsubsection{Fungi biological isolation}

Fungi were isolated from stored organic and conventional roots. They were sampled from roots harvested and stored in 2009. Five roots from each sample were thoroughly rinsed under running tap water and two $15 \mathrm{~mm}$ cylinders centred through the root were removed from each root. The cylinders were surface disinfected $10 \mathrm{~s}$ in $70 \%$ ethanol, transferred to $2.5 \% \mathrm{NaOCl}$ for $3 \mathrm{~min}$ and finally rinsed three times in sterile water. The disinfected cylinders were cut longitudinally and the outermost $(1 \mathrm{~mm})$ periderm layer cut off. The root pieces were placed on potato dextrose agar (PDA) and on malt extract agar (ME) both media were amended with Novobiocin $(250 \mathrm{mg} / \mathrm{L})$ and incubated at $16^{\circ} \mathrm{C}$ and $20^{\circ} \mathrm{C}$. Fungi growing from the rotted tissue were purified on PDA. Fourteen fungi differing in colony morphology and spore morphology, seven of the isolates were non-sporulating, were selected for molecular identification based on ITS1-5.8s-ITS2 sequencing. For DNA isolation, the fungi were grown in potato dextrose broth at $100 \mathrm{rpm}$ at room temperature for 14 days. The fungal biomass was harvested on nylon mesh $(32 \mu \mathrm{m})$ and rinsed with sterilised water. The biomass was frozen at $-80^{\circ} \mathrm{C}$ and subsequently freeze-dried. DNA was extracted using Qiagen DNAeasy kit (Qiagen, Valencia, USA). The total reaction volume of the PCR reaction was $20 \mu \mathrm{L}$ and contained $1 \times$ Taq polymerase buffer including $1.5 \mathrm{mM} \mathrm{MgCl}_{2}, 0.3 \mathrm{mM}$ of each dNTP, $0.5 \mu \mathrm{M}$ each of the primers ITS1 and ITS4 (White et al., 1990), and 2.5U Taq DNA polymerase (Amplicon, Copenhagen, DK). The PCR amplification was performed as described by Sundelin et al. (2009), except for the use of a Mastercycler personal (Eppendorf) PCR machine. For purifying PCR products: $5 \mu \mathrm{L}$ PCR mixture was mixed with $10 \mathrm{U}$ Exonuclease I, E. coli and $1 \mathrm{U}$ FastAP Thermosensitive Alkaline Phosphatase (Fermentas, Helsingborg, Sweden) and incubated $15 \mathrm{~min}$ at $37^{\circ} \mathrm{C}$ followed by $15 \mathrm{~min}$ incubation at $85^{\circ} \mathrm{C}$ to stop the reaction. Sequencing and query search of the PCR products was performed as described above.

\subsection{Statistical methods}

The micromycota data were compared between the cropping systems, the comparison was made using the Mann-Whitney test for nonparametric testing. The statistics of the proteomic experiment (the Student's $t$-test) was performed using Image Master ${ }^{\mathrm{TM}}$ 2D Platinum software v.5.0 (GE Healthcare).

\section{Results}

\subsection{Proteomics}

The coverage of the proteome is approximately 1200 spots. The intensity of 10 spots increased and 5 decreased in response to cold storage $(p<0.05$ and fold $\geq 1.5)$ in all the three replicates and both in the organic and conventional root (Table 1 and Fig. 2). No significant differences were observed between organic and conventional roots.

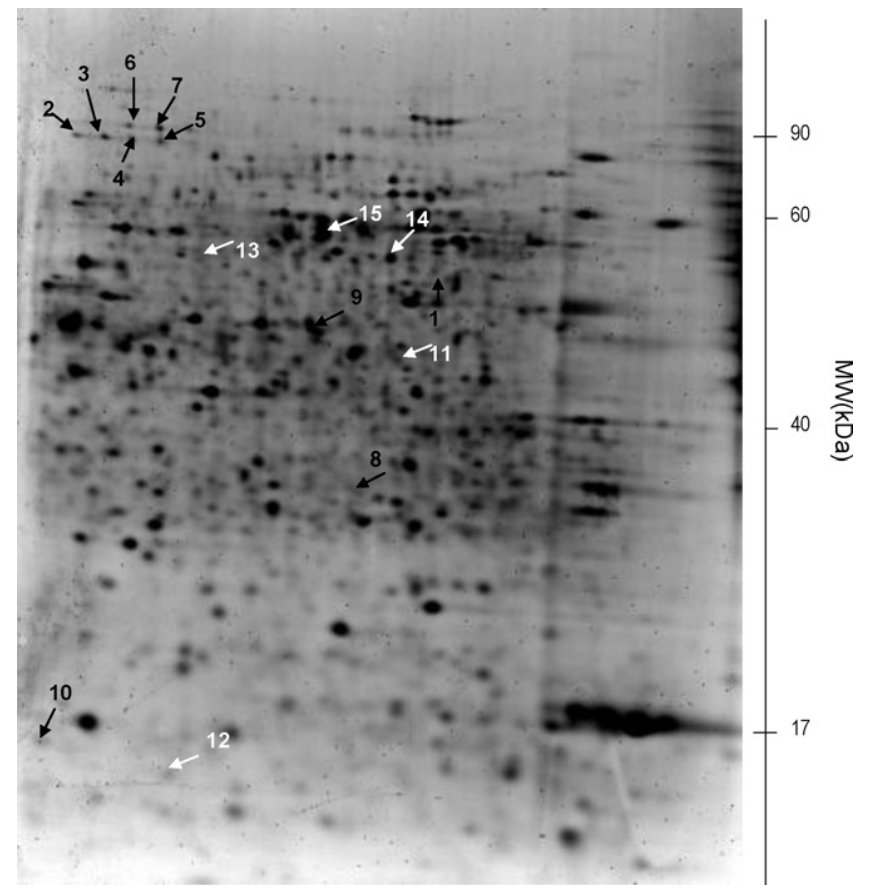

Fig. 2. Representative gel of carrot root (pI 4 to 7). The protein spots numbered in black are those that increased significantly during the storage and those in white decreased. The numbers in the gel images refer to protein numbers in Table 2. 
Table 1

The effects of cold storage on the proteome of organically and conventionally cultivated carrots. Analysis of 2D-PAGE gels for proteins exhibiting more than 1.5 fold alteration in relative intensity following storage.

\begin{tabular}{|c|c|c|c|c|c|c|c|c|c|c|c|c|c|}
\hline \multirow[t]{2}{*}{$\begin{array}{l}\text { Spot } \\
\text { number }\end{array}$} & \multicolumn{3}{|c|}{$\begin{array}{l}\text { Fold changes in the conventional } \\
\text { roots (in the organic roots) }\end{array}$} & \multirow{2}{*}{$\begin{array}{l}\text { Theoritical/ } \\
\text { observed MW } \\
\text { (kDa) }\end{array}$} & \multirow[t]{2}{*}{$\begin{array}{l}\text { Theoritical/ } \\
\text { observed pl }\end{array}$} & \multirow[t]{2}{*}{$\begin{array}{l}\text { Accession } \\
\text { number }^{b}\end{array}$} & \multirow[t]{2}{*}{ Protein name $^{c}$} & \multirow[t]{2}{*}{ Organism } & \multirow{2}{*}{$\begin{array}{l}\text { Biological } \\
\text { process } \\
\text { (molecular }^{\text {function)d }}\end{array}$} & \multirow[t]{2}{*}{$\begin{array}{l}\text { Matched } \\
\text { peptides }\end{array}$} & \multirow[t]{2}{*}{$\begin{array}{l}\text { Sequence } \\
\text { coverage (\%) }\end{array}$} & \multirow[t]{2}{*}{ Score } & \multirow[t]{2}{*}{ Sequence $^{\mathrm{f}}$} \\
\hline & $\begin{array}{l}\begin{array}{l}\text { Harvest/11 } \\
\text { month }\end{array}\end{array}$ & $\begin{array}{l}\text { Harvest } / 4 \\
\text { months }\end{array}$ & $\begin{array}{l}\text { Harvest } / 6 \\
\text { months }\end{array}$ & & & & & & & & & & \\
\hline \multicolumn{14}{|c|}{ Spot intensity increasing } \\
\hline \multirow[t]{7}{*}{1} & $1.9(1.7)$ & $2.3(2.1)$ & $2.3(2.2)$ & $51.187 / 49.902$ & $5.52 / 5.28$ & GI:78099188 & $\begin{array}{l}\text { Probable } \\
\text { L-ascorbate } \\
\text { peroxidase } 8\end{array}$ & $\begin{array}{l}\text { Oryza sativa subsp. } \\
\text { japonica }\end{array}$ & $\begin{array}{l}\text { Stress response } \\
\text { (hydrogen } \\
\text { peroxide } \\
\text { removal) }\end{array}$ & 7 & $26 \%$ & 147 & $\begin{array}{l}\text { YPGITYADLFQLASATAIEE } \\
\text { AGGPKIPMK }\end{array}$ \\
\hline & & & & & & & & & & & & 59 & YGRVDVTAAEQCPPEGR \\
\hline & & & & & & & & & & & & 40 & EVFYRMGLDDK (Mox) \\
\hline & & & & & & & & & & & & 65 & MGLDDKEIVALSGAHTLGR (Mox) \\
\hline & & & & & & & & & & & & 71 & FDNSYFKDIK \\
\hline & & & & & & & & & & & & 98 & DQDLLVLPTDAALFEDPSFK \\
\hline & & & & & & & & & & & & 102 & LSDLGAKFDPPEGFSLDDE PAVEEK \\
\hline \multirow[t]{4}{*}{2} & $1.6(1.5)$ & $1.8(1.9)$ & $2.1(2)$ & $84.589 / 83.978$ & $6.51 / 6.77$ & GI:8134569 & $\begin{array}{l}\text { Methionine } \\
\text { synthase }\end{array}$ & $\begin{array}{l}\text { Solenostemon } \\
\text { scutellarioides }\end{array}$ & $\begin{array}{l}\text { Amino acid } \\
\text { biosynthesis } \\
\text { (methyl } \\
\text { transfer) }\end{array}$ & 4 & $6 \%$ & 44 & YLFAGVVDGR \\
\hline & & & & & & & & & & & & 38 & AGITVIQIDEAALR \\
\hline & & & & & & & & & & & & 14 & SDEKLLSVFR \\
\hline & & & & & & & & & & & & 71 & YGAGIGPGVYDIHSPR \\
\hline \multirow[t]{5}{*}{3} & $2.1(2.2)$ & $2.5(2.4)$ & $2.5(2.4)$ & $84.589 / 83.981$ & $6.51 / 6.659$ & GI:8134569 & $\begin{array}{l}\text { Methionine } \\
\text { synthase }\end{array}$ & S. scutellarioides & $\begin{array}{l}\text { Amino acid } \\
\text { biosynthesis } \\
\text { (methyl } \\
\text { transfer) }\end{array}$ & 5 & $6 \%$ & 76 & YLFAGVVDGR \\
\hline & & & & & & & & & & & & 93 & AGITVIQIDEAALR \\
\hline & & & & & & & & & & & & 53 & SDEKLLSVFR \\
\hline & & & & & & & & & & & & 14 & LLSVFR \\
\hline & & & & & & & & & & & & 64 & YGAGIGPGVYDIHSPR \\
\hline \multirow[t]{3}{*}{4} & $1.7(1.5)$ & $2.1(1.9)$ & $2.4(2.5)$ & $84.821 / 84.459$ & $6.27 / 6.58$ & GI:8134568 & $\begin{array}{l}\text { Methionine } \\
\text { synthase }\end{array}$ & $\begin{array}{l}\text { Mesembryanthemum } \\
\text { crystallinum }\end{array}$ & $\begin{array}{l}\text { Amino acid } \\
\text { biosynthesis } \\
\text { (methyl } \\
\text { transfer) }\end{array}$ & 4 & $7 \%$ & 54 & YLFAGVVDGR \\
\hline & & & & & & & & & & & & 56 & KLNLPILPTTTIGSFPQTVELR \\
\hline & & & & & & & & & & & & 25 & SDEKLLSVFR \\
\hline \multirow[t]{3}{*}{5} & $1.5(1.5)$ & $1.7(1.8)$ & $1.7(1.8)$ & $84.821 / 84.528$ & $6.27 / 6.41$ & GI:8134568 & $\begin{array}{l}\text { Methionine } \\
\text { synthase }\end{array}$ & M. crystallinum & $\begin{array}{l}\text { Amino acid } \\
\text { biosynthesis } \\
\text { (methyl } \\
\text { transfer) }\end{array}$ & 4 & $4 \%$ & $\begin{array}{l}51 \\
68\end{array}$ & $\begin{array}{l}\text { YGAGGGGVYDIHSPR } \\
\text { YLFAGVVDGR }\end{array}$ \\
\hline & & & & & & & & & & & & 34 & SDEKLLSVFR \\
\hline & & & & & & & & & & & & 20 & LLSVFR \\
\hline \multirow[t]{3}{*}{6} & $1.6(1.7)$ & $2(1.9)$ & $2(1.9)$ & $84.589 / 84.135$ & $6.51 / 6.53$ & GI:8134569 & $\begin{array}{l}\text { Methionine } \\
\text { synthase }\end{array}$ & S. scutellarioides & $\begin{array}{l}\text { Amino acid } \\
\text { biosynthesis } \\
\text { (methyl } \\
\text { transfer) }\end{array}$ & 4 & $5 \%$ & $\begin{array}{l}59 \\
66\end{array}$ & $\begin{array}{l}\text { YGAGIGPGVYDIHSPR } \\
\text { YLFAGVVDGR }\end{array}$ \\
\hline & & & & & & & & & & & & 91 & AGITVIQIDEAALR \\
\hline & & & & & & & & & & & & $\begin{array}{l}55 \\
18\end{array}$ & $\begin{array}{l}\text { SDEKLLSVFR } \\
\text { LLSVFR }\end{array}$ \\
\hline \multirow[t]{3}{*}{7} & $1.6(1.7)$ & $2(1.9)$ & $2(1.9)$ & $84.589 / 83.8857$ & $6.51 / 6.42$ & GI:8134569 & $\begin{array}{l}\text { Methionine } \\
\text { synthase }\end{array}$ & S. scutellarioides & $\begin{array}{l}\text { Amino acid } \\
\text { biosynthesis } \\
\text { (methyl } \\
\text { transfer) }\end{array}$ & 4 & $6 \%$ & 71 & YLFAGVVDGR \\
\hline & & & & & & & & & & & & $\begin{array}{l}85 \\
52\end{array}$ & $\begin{array}{l}\text { AGITVIOIDEAALR } \\
\text { SDEVLISVFR }\end{array}$ \\
\hline & & & & & & & & & & & & $\begin{array}{l}52 \\
68\end{array}$ & $\begin{array}{l}\text { SDEKLLSVFR } \\
\text { YGAGIGPGVYDIHSPR }\end{array}$ \\
\hline
\end{tabular}




\begin{tabular}{|c|c|c|c|c|c|c|c|c|c|c|c|c|c|}
\hline \multirow[t]{2}{*}{$\begin{array}{l}\text { Spot } \\
\text { number }\end{array}$} & \multicolumn{3}{|c|}{$\begin{array}{l}\text { Fold changes in the conventional } \\
\text { roots (in the organic roots) }\end{array}$} & \multirow{2}{*}{$\begin{array}{l}\text { Theoritical/ } \\
\text { observed MW } \\
\text { (kDa) }\end{array}$} & \multirow[t]{2}{*}{$\begin{array}{l}\text { Theoritical/ } \\
\text { observed pI }\end{array}$} & \multirow[t]{2}{*}{$\begin{array}{l}\text { Accession } \\
\text { number }\end{array}$} & \multirow[t]{2}{*}{ Protein name $\mathrm{e}^{\mathrm{c}}$} & \multirow[t]{2}{*}{ Organism } & \multirow{2}{*}{$\begin{array}{l}\text { Biological } \\
\text { process } \\
\text { (molecular } \\
\text { function) }\end{array}$} & \multirow[t]{2}{*}{$\begin{array}{l}\text { Matched } \\
\text { peptides }\end{array}$} & \multirow[t]{2}{*}{$\begin{array}{l}\text { Sequence } \\
\text { coverage (\%) }\end{array}$} & \multirow[t]{2}{*}{ Score } & \multirow[t]{2}{*}{ Sequence $^{\mathrm{f}}$} \\
\hline & $\begin{array}{l}\text { Harvest/1 } \\
\text { month }\end{array}$ & $\begin{array}{l}\text { Harvest } / 4 \\
\text { months }\end{array}$ & $\begin{array}{l}\text { Harvest } / 6 \\
\text { months }\end{array}$ & & & & & & & & & & \\
\hline 8 & $2.2(2)$ & $2.6(2.5)$ & $2.6(2.5)$ & $30261 / 29745$ & $5.66 / 5.84$ & GI:3668173 & $\begin{array}{l}\text { Vegetative } \\
\text { storage protein }\end{array}$ & Arabidopsis thaliana & Storage & 2 & $13 \%$ & 65 & AYVEDYLITSK \\
\hline 9 & $1.7(1.9)$ & $2.2(2.2)$ & $2.2(2.2)$ & $47972 / 47854$ & $5.57 / 5.29$ & GI:90110845 & Enolase & O. sativa & Glycolysis & 3 & $12 \%$ & $\begin{array}{r}104 \\
74 \\
81 \\
95\end{array}$ & $\begin{array}{l}\text { NDTVNVWIFDLDDTLLSSIPYYAK } \\
\text { AVDNVNSVIAPALIGK } \\
\text { LGANAILAVSLAICK }\end{array}$ \\
\hline $\begin{array}{l}10 \\
\text { Spot intens }\end{array}$ & $\begin{array}{c}1.6(1.8) \\
y \text { decreasing }\end{array}$ & $1.9(2)$ & $1.9(2)$ & $15356 / 15136$ & $8.00 / 6.80$ & GI:57506540 & Dehydrin & Daucus carota & Stress response & 1 & $11 \%$ & 102 & SGSSSSSSSEDDGMGGR (Mox) \\
\hline \multirow[t]{4}{*}{11} & $1.6(1.5)$ & $1.6(1.5)$ & $1.8(1.7)$ & $41735 / 41443$ & $5.49 / 5.69$ & GI:1703108 & Actin 7 & A. thaliana & $\begin{array}{l}\text { Cytoskeleton } \\
\text { (structural } \\
\text { molecule } \\
\text { activity) }\end{array}$ & 5 & $14 \%$ & 59 & VAPEEHPVLLTEAPLNPK \\
\hline & & & & & & & & & & & & 22 & LDLAGRDLTDSLMK \\
\hline & & & & & & & & & & & & $\begin{array}{r}16 \\
109\end{array}$ & $\begin{array}{l}\text { LDLAGRDLTDSLMK (Mox) } \\
\text { SSSSVEKNYELPDGQVITIG AER }\end{array}$ \\
\hline & & & & & & & & & & & & 76 & NYELPDGQVITIGAER \\
\hline \multirow[t]{2}{*}{12} & $1.9(1.6)$ & $2.2(2.1)$ & $2.2(2.1)$ & $16.547 / 15.258$ & $6.30 / 6.54$ & GI:1346675 & $\begin{array}{l}\text { Nucleoside } \\
\text { diphosphate } \\
\text { kinase } 1\end{array}$ & A. thaliana & $\begin{array}{l}\text { Amino acid } \\
\text { biosynthesis } \\
\text { (role in } \\
\text { synthesis of } \\
\text { nucleoside } \\
\text { triphosphates) }\end{array}$ & 3 & $17 \%$ & 119 & KIIGATNPAESAPGTIR \\
\hline & & & & & & & & & & & & $\begin{array}{r}130 \\
64\end{array}$ & $\begin{array}{l}\text { IIGATNPAESAPGTIR } \\
\text { GDFAIDIGR }\end{array}$ \\
\hline \multirow[t]{2}{*}{13} & $1.5(1.6)$ & $1.8(1.8)$ & $1.8(1.8)$ & $50.733 / 49.821$ & $4.8 / 4.74$ & GI:267073 & Beta-tubulin & A. thaliana & $\begin{array}{l}\text { Cytoskeleton } \\
\text { (structural } \\
\text { molecule } \\
\text { activity) }\end{array}$ & 3 & $9 \%$ & 114 & PDNFVFGQSGAGNNWAK \\
\hline & & & & & & & & & & & & $\begin{array}{l}49 \\
82\end{array}$ & $\begin{array}{l}\text { FPGQLNSDLR } \\
\text { NSSYFVEWIPNNVK }\end{array}$ \\
\hline 14 & $1.8(1.6)$ & $2.3(2.2)$ & $2.3(2.2)$ & $49.823 / 49.752$ & $5.19 / 5.62$ & GI:6094430 & Alpha-tubulin & Eleusine indica & $\begin{array}{l}\text { Cytoskeleton } \\
\text { (structural } \\
\text { molecule } \\
\text { activity) }\end{array}$ & 2 & $7 \%$ & 56 & LISQVISSLTTSLR \\
\hline 15 & $2.5(2.4)$ & $2.7(2.6)$ & $2.7(2.6)$ & $53.029 / 50.854$ & $6 / 5.78$ & GI:122239967 & $\begin{array}{l}\text { Ribulose } \\
\text { bisphosphate } \\
\text { carboxylase }\end{array}$ & D. carota & $\begin{array}{l}\text { Photosynthesis } \\
\text { (photorespira- } \\
\text { tion) }\end{array}$ & 2 & $4 \%$ & $\begin{array}{l}70 \\
53\end{array}$ & $\begin{array}{l}\text { FDGAINVDITEFQTNLVPYPR } \\
\text { DTDILAAFR }\end{array}$ \\
\hline
\end{tabular}

a Protein spot number as in Fig.

b Protein accession number derive from NCBI database.

c Protein name derived from either Swissprot or NCBI databases.

d Biological process the proteins is involved in and molecular function derived from either Swissprot or NCBI databases.

e Ions mascot score for identified peptides by MSMS at $p \leq 0.05$.

f Mox: oxidized methionine. 
Table 2

List of the fungal taxa identified from ITS2 ribosomal DNA sequences of fungi cloned from samples of organic and conventional grown carrots in 2007 and 2008 . Roots were cold stored at $1{ }^{\circ} \mathrm{C}$ for one month before sampling.

\begin{tabular}{|c|c|c|c|c|c|}
\hline \multirow[t]{2}{*}{ Taxa } & \multirow[t]{2}{*}{ Maximal identity (\%) } & \multicolumn{2}{|l|}{2007} & \multicolumn{2}{|l|}{2008} \\
\hline & & Organic \% & Conventional \% & Organic \% & Conventional \% \\
\hline Leptodontidium orchidicola & $98-100$ & 9 & 10 & 18 & 15 \\
\hline Cadophora luteo-olivacea & 100 & 3 & 1 & 2 & - \\
\hline Tetracladium spp. & $97-99$ & 7 & 4 & - & - \\
\hline Tetracladium maxilliforme & $98-100$ & 24 & 19 & 13 & 17 \\
\hline Tetracladium furcatum & $97-100$ & 10 & 10 & 11 & 4 \\
\hline Peyronellaea spp./Phoma spp. ${ }^{a}$ & 100 & 0 & 8 & 2 & - \\
\hline Phoma eupyrena/P.herbarum & $99-100$ & 6 & 5 & 10 & 2 \\
\hline Epicoccum sorghinum/Phoma spp. & 100 & 3 & 3 & - & - \\
\hline Davidiella ssp./Cladosporium ssp. & 100 & - & 1 & 3 & 6 \\
\hline Ilyonectria macrodidyma & $99-100$ & 3 & - & 5 & 8 \\
\hline Ilyonectria macrodidyma/I. radicicola & $98-99$ & - & - & 2 & 2 \\
\hline Ilyonectria radicola/Ilyonectria spp.b & 100 & - & 1 & 7 & 6 \\
\hline Giberella avenacea/Fusarium tricinctum & 99 & 3 & 4 & 2 & 8 \\
\hline Gibberella moniliformis/G. thapsina & 100 & 3 & - & - & - \\
\hline Bipolaris sp./Curvularia clavata & $98-100$ & 10 & 7 & - & - \\
\hline Pyrenochaeta sp. & 100 & 1 & 1 & 2 & 4 \\
\hline Dendryphion nanum & 100 & - & - & 3 & 2 \\
\hline Geomyces sp. & 96 & - & - & - & 6 \\
\hline Geomyces pannorum & 100 & - & 1 & 2 & 4 \\
\hline Geomyces leuteus & 100 & - & - & - & 4 \\
\hline Glomerella acutata $^{\mathrm{c}}$ & $99-100$ & - & 7 & 2 & 2 \\
\hline Rhexocercosporidium sp. & 98 & - & - & 3 & - \\
\hline Rhexocercosporidium carotae & $99-100$ & 4 & 7 & - & 6 \\
\hline Marssonina brunnea & 83 & 3 & - & - & - \\
\hline Apiognomonia errabunda & 100 & - & 1 & 2 & 2 \\
\hline Dothideomycetes & 93 & - & 3 & - & - \\
\hline Ascomycota $^{\mathrm{d}}$ & $86-100$ & 11 & 5 & 11 & 6 \\
\hline Number of isolates & & $n=70$ & $n=73$ & $n=61$ & $n=53$ \\
\hline
\end{tabular}

a Significant difference $(p<0.05)$ in recovery of Peyronellaea spp./Phoma spp. between the cropping systems in 2007 using the Mann-Witney test. No other comparisons differed significantly.

b Best hits included either I. rufa, I. crassa or I. robusta all having the same maximal identity as the dominating hits of Ilyonectria radicicola.

c For 3 isolates the blast search could not distinguish G. acutata from G. fioriniae.

d Sequences of ascomycetes only occurring once in 2007 and 2008 were identified to the following taxa: Aureobasidium pullulans, Fusarium equiseti, Paraphoma chrysanthemicola, Aspergillus spp., Zalerion varium, Cochliobolus lunatus, Leptosphaeria sp., Cadophora sp, Glarea sp, Penicillium spp., Spirosphaera cupreorufescens, Epicoccum nigrum, Cladosporium sphaerospermum/C. cladosporioides, Fusarium merismoides, Exophiala sp., Heliotiales.

Only two of the 15 proteins identified matched directly to carrot protein sequences in the databases. Most of the proteins identified were at their predicted position in the gels (based on MW and $\mathrm{pI}$ ) but some of the identified proteins are inferred to be degraded since their positions in the gels did not correspond to the theoretical pI or MW (Table 1 and Fig. 2). All spot identifications in Table 1 are based on two or more peptides except for spot 10 which is based on a single peptide: it is conceivable that this therefore represents contamination of the major spot from a protein in the background which is unaffected by cold storage. Among the 15 identified proteins, three of them are part of the cytoskeleton (spots 11,13 and 14 ), and three others are involved in stress responses (spot 1, 10 and 12).

\subsection{Micromycota}

In the roots harvested in 2007,143 different fungal isolates were identified as belonging to 22 taxa and 114 isolates identified to 21 taxa in the roots from 2008 (Table 2). The occurrence of each fungal taxon in the organic and conventional root were compared. In 2007, only Peyronellaea spp./Phoma spp. differed significantly in occurrence; this taxon occurred more often in the conventional roots than in the organic ones. No significant difference could be attributed to the cropping systems in 2008. Representatives from the nine most frequently occurring taxa were identified in both years (Fig. 2) and from most samples. This reproducibility supports the conclusion that these data are robust (see supplementary Tables 1 and 2). However, frequencies of occurrence in our samples varied considerably, e.g., 7\% Geomyces was found in 2008 against $1 \%$ in 2007 and the same was seen for Davidiellaceae. On the other hand, many of the other groups like Tetracladium, DSE, Pleosporaceae, Nectriaceae and Didymellaceae were consistently represented with high frequencies in both years. Averaged over year and cropping system, Tetracladium, Leptodontidium, Nectriaceae and Didymellaceae were the most frequently isolated taxa, respectively, representing $31 \%, 14 \%, 13 \%$ and $10 \%$ of all isolates.

Culturable fungi were isolated from stored roots in 2009. Based on ITS 1-2 sequences, the following taxa were identified Tetracladium furcatum, Tetracladium maxilliforme, Leptodotidium sp., Ilyonectria spp., Fusarium solani, Plectosphaerella cucumerina (syn. Fusarium tabacinum), Phoma spp., Pyrenochaeta sp., Acremonium strictum. All the fungi identified from biological isolation were Ascomycetes.

\section{Discussion}

We have demonstrated in this study, using two completely independent methods to monitor physiological quality, that no substantial differences have arisen between organic and conventionally cultivated carrots during storage.

At the protein level, three proteins that changed significantly during storage are involved in stress responses. The increase of ascorbate peroxidase (spot 1) correlated with its involvement in response to abiotic and biotic stress; this protein has been reported previously to accumulate following oxidative stress in roots due to cold treatment (Neilson et al., 2010). Interestingly, enhanced peroxidase activity might have an influence on bacterial as well as fungal endophyte populations. A dehydrin (spot 10) also accumulated and has been reported previously to be induced by drought, 
salinity or/and low temperature (Close, 1996) and might stabilize cell membranes and protect other proteins from denaturation during dehydration (Allagulova et al., 2003). Dehydrin accumulation has been shown in proteomic studies of cold stress in Arabidopsis (Kawamura and Uemura, 2003; Renaut et al., 2004). It might also play a role in conjunction with molecular chaperones, DNA binding and repair, or participate in the cytoskeleton (Rahman et al., 2011). Tubulins (alpha and beta) are the major constituents of microtubules. Both alpha- and beta- tubulin levels (spots 13 and 14) decreased during cold storage. The reduced abundance of tubulins correlates with the appearance of cold-stable microtubules which is linked with low temperature stress (Abdrakhamanova et al., 2003). Actin 7 (spot11), another cytoskeleton component decreased during storage. The cytoskeletal proteins assemble into polymers that play an important role in the cell structure and are determinant of the cell shape; during cold adaptation, a reorganisation of these polymers is necessary to preserve the cell integrity. The presence of RuBisCo (spot15) in roots is surprising, because this is a non-photosynthetic organ but it has previously been reported in roots (Suzuki et al., 2009; Louarn et al., 2012). $\mathrm{A} \mathrm{CO}_{2}$-binding role for RuBisCo has been demonstrated in developing Brassica napus embryos (Schwender et al., 2004). RuBisCo might have a similar function in roots with respect to the expression of decarboxylating enzymes of the Krebs cycle (Mooney et al., 2006). A decrease of RuBisCo could indicate a slowing down of the energy metabolism. Vegetative storage protein (spot 8) levels increased and suggests an adaptation of the roots in order to store energy for the next year; carrot is a biannual crop plant and can thus withstand a period of cold/freezing temperatures over winter.

The fungal communities of cold-stored carrot roots were identified by rDNA clone libraries that enabled us to identify 257 Ascomycetes isolates (clones) in the roots. The FITS PCR-primers used exhibit greatest affinity for detection of Ascomycetes and would not be expected to yield Basidiomycetes or Glomeromycetes due to mismatches at the primer binding sites (Fig. 1). Although the FITS reverse primer has a single mismatch at the $5^{\prime}$ end for several Ascomycete genera, it does not seem to affect the efficiency of the primers to amplify, e.g., Tetracladium and Leptodontidium. The robustness of the DNA based method was confirmed by the result of the biological isolation that yielded solely Ascomycete taxa, all bar one was also was detected by the FITS primers. In general, ascomycetes also seem to constitute the majority of endophytic populations in roots of other species studied (Upson et al., 2009; Andrade-Linares et al., 2011). Concerning the absence of Glomeromycetes, these are known to be associated with carrots but most frequently with the fine roots which were not sampled in this study (Dumas-Gaudot et al., 2004). There was no significant effect of the cropping system on the micromycota community, only Peyronellaea spp./Phoma spp. significantly differ (Table 2). In contrast Wilberforce et al. (2003) found that agricultural management practice affected communities of root-endophytic fungi in grasslands at different locations (Wilberforce et al., 2003). However, in our field experiment, the plots were situated at the same location.

The nine most frequently occurring taxa were identified in both years but the isolation frequencies of clones varied considerably between 2007 and 2008 (Fig. 2). These differences could be related to the relatively small sample size (257 clones), the varying climatic conditions or the use of different seed lots in the two years. The occurrence of several taxa only once, either in 2007 or in 2008, can be attributed to the same factors (Table 2). These pathogens can survive inside carrot roots (or carrot debris) in the field and might become inoculum sources that will infect a later crop. The taxon Leptodontidium orchidicola is frequent in both years and Cadophora spp., belong to the artificial group "dark septate endophytes" (DSE) which occur widely in association with plant roots in cold stressed habitats (Upson et al., 2009; Rosa et al., 2010) (Table 2). The DSE
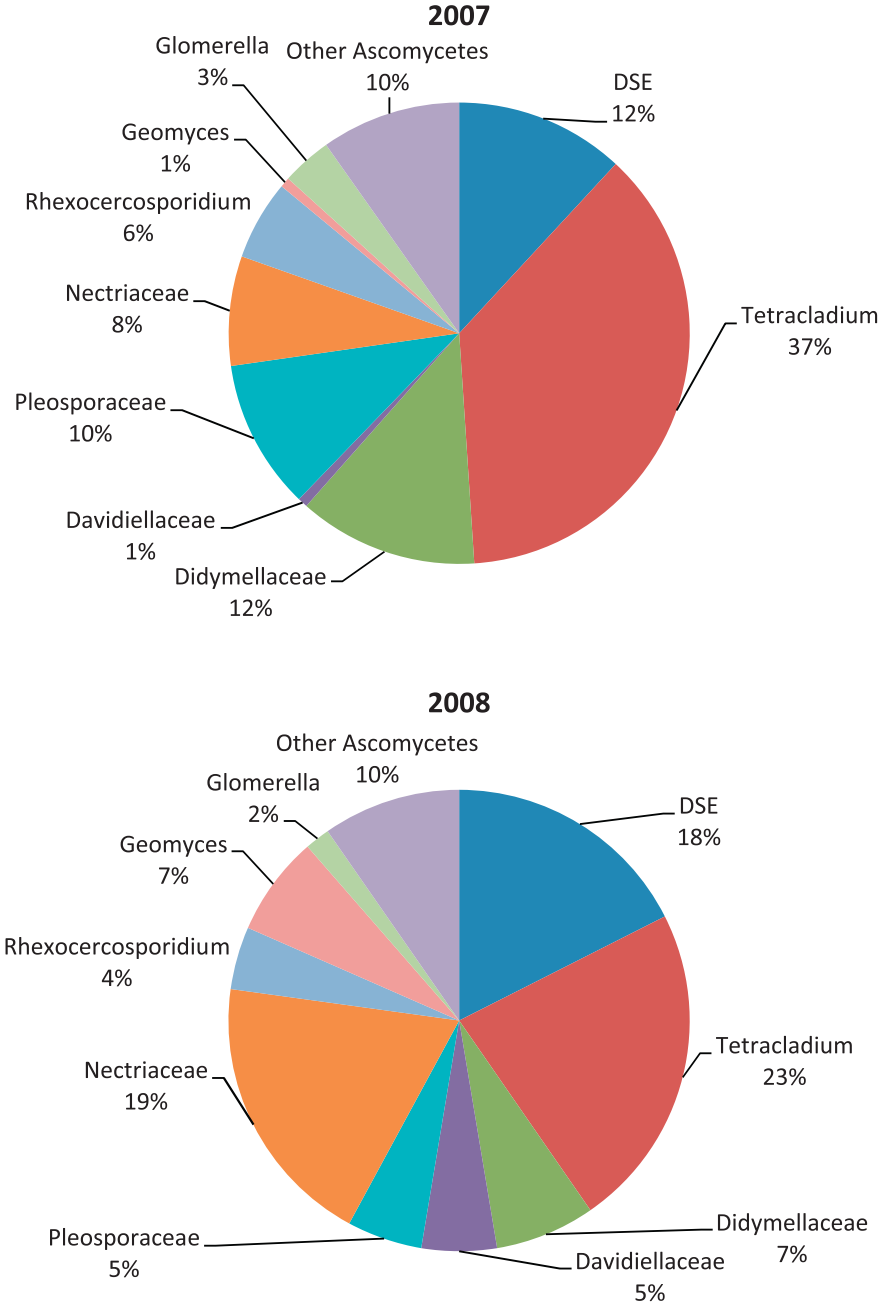

Fig. 3. Proportion of fungal taxa recovered from DNA sequence analysis of cold stored carrots in 2007 and in 2008. DSE (dark septate fungal endophytes) contains Leptodontidium and Cadophora. Nectriaceae contains Fusarium, Giberella and Ilyonectria. Pleosporaceae contains Bipolaris, Pyrenochaeta and Dendryphion. Davidiellaceae contains Davidiella and Cladosporium and, Didymellaceae contains Phoma and Peyronella.

have received much attention since they can stimulate host growth and protect plants against various stress conditions, e.g., by secretion of secondary metabolites (Schulz et al., 2002; Porras-Alfaro and Bayman, 2011). Other taxa Geomyces spp., and Davidiella spp. detected in the cold-stored carrots have also been identified as endophytes in roots of plant species adapted to cold conditions (Rosa et al., 2010) (Fig. 3).

Some of the identified taxa, e.g., Rhexocercosporidium carotae (Kastelein et al., 2007) and Fusarium spp., are known as carrot postharvest pathogens. However, they remained latent as no visible symptoms developed during the 6 months of cold storage (Table 2). This could be due to defence mechanisms like the accumulation of the antioxidative ascorbate peroxidase which can protect against necrotrophs (Table 1) induced either by the cold environment or by endophytic species.

The changes observed at the protein level indicate an adaptation of the roots to the cold storage conditions within the first month of storage after which the root proteome was stable for the remaining 5 months. Both organic and conventional roots were still marketable after 6 months of cold storage. The observed changes of the proteome could be associated with a cold adaptation of the carrots. We presumably did not isolate all the fungal endophytes taxa but with over 257 clones identified, representing 
approximately 40 taxa from a total of 72 carrots, we believe these samples are representative of the micromycota. The fact that some pathogens were identified as symptomless endophytes might be due to activation of defence mechanisms induced either by the cold environment or certain endophytes. We conclude that cropping system did not have an influence on the postharvest quality of the carrots during six months of cold storage.

\section{Acknowledgements}

We thank Karin Olesen for her expert assistance during the sample collection and the micromycota experiments, and Andrea Maria Lorentzen for mass spectrometry support. This project was supported by an International Centre for Research in Organic Food Systems (ICROFS) grant "Organic cropping Systems for Vegetable production, - product Quality, Natural Regulation and Environmental effects (VEG-QURE 3304-FOJO-05-45-07)".

\section{Appendix A. Supplementary data}

Supplementary data associated with this article can be found, in the online version, at http://dx.doi.org/10.1016/j.postharvbio. 2012.08.011.

\section{References}

Abdrakhamanova, A., Wang, Q.Y., Khokhlova, L., Nick, P., 2003. Is microtubule disassembly a trigger for cold acclimation? Plant and Cell Physiology 44, 676-686.

Allagulova, C., Gimalov, F.R., Shakirova, F.M., Vakhitov, V.A., 2003. The plant dehydrins: structure and putative functions. Biochemistry (Moscow) 68, 945-951.

Andrade-Linares, D., Grosch, R., Restrepo, S., Krumbein, A., Franken, P., 2011. Effects of dark septate endophytes on tomato plant performance. Mycorrhiza 21, 413-422.

Botella, L., Diez, J., 2011. Phylogenic diversity of fungal endophytes in Spanish stands of pinus halepensis. Fungal Diversity 47, 9-18.

Close, T.J., 1996. Dehydrins: emergence of a biochemical role of a family of plant dehydration proteins. Physiologia Plantarum 97, 795-803.

D’Amico, M., Frisullo, S., Cirulli, M., 2008. Endophytic fungi occurring in fennel, lettuce, chicory, and celery - commercial crops in southern Italy. Mycological Research 112, 100-107.

Degand, H., Faber, A.M., Dauchot, N., Mingeot, D., Watillon, B., Van Cutsem, P., Morsomme, P., Boutry, M., 2009. Proteomic analysis of chicory root identifies proteins typically involved in cold acclimation. Proteomics 9, 2903-2907.

Dumas-Gaudot, E., Valot, B., Bestel-Corre, G., Recorbet, G., St-Arnaud, M., Fontaine, B., Dieu, M., Raes, M., Saravanan, R.S., Gianinazzi, S., 2004. Proteomics as a way to identify extra-radicular fungal proteins from Glomus intraradices - RiT-DNA carrot root mycorrhizas. FEMS Microbiology Ecology 48, 401-411.

Farrar, J.J., Pryor, B.A., Davis, R.M., 2004. Alternaria diseases of carrot. Plant Disease 88, 776-784.

Gazis, R., Chaverri, P., 2010. Diversity of fungal endophytes in leaves and stems of wild rubber trees (Hevea brasiliensis) in Peru. Fungal Ecology 3, 240-254.

Griffith, M., Lumb, C., Wiseman, S.B., Wisniewski, M., Johnson, R.W., Marangoni, A.G., 2005. Antifreeze proteins modify the freezing process in planta. Plant Physiology 138, 330-340.

Griffith, M., Yaish, M.W.F., 2004. Antifreeze proteins in overwintering plants: a tale of two activities. Trends in Plant Science 9, 399-405.

Higgins, K.L., Arnold, A.E., Miadlikowska, J., Sarvate, S.D., Lutzoni, F., 2007. Phylogenetic relationships, host affinity, and geographic structure of boreal and arctic endophytes from three major plant lineages. Molecular Phylogenesis Evolution 42, 543-555.

Jayaraj, J., Rahman, M., Wan, A., Punja, Z.K., 2009. Enhanced resistance to foliar fungal pathogens in carrot by application of elicitors. Annals of Applied Biology 155, 71-80.

Kastelein, P., Stilma, E.S.C., Elderson, J., Kohl, J., 2007. Occurrence of Rhexocercosporidium carotae on cold stored carrot roots in the Netherlands. European Journal of Plant Pathology 117, 293-305.

Kawamura, Y., Uemura, M., 2003. Mass spectrometric approach for identifying putative plasma membrane proteins of Arabidopsis leaves associated with cold acclimation. Plant Journal 36, 141-154.

Kernaghan, G., Sigler, L., Khasa, D., 2003. Mycorrhizal and root endophytic fungi of containerized Picea glauca seedlings assessed by rDNA sequence analysis. Microbial Ecology 45, 128-136.

Kora, C., McDonald, M.R., Boland, G.J., 2005. Occurrence of fungal pathogens of carrots on wooden boxes used for storage. Plant Pathology 54, 665-670.

Kuldau, G., Bacon, C., 2008. Clavicipitaceous endophytes: their ability to enhance resistance of grasses to multiple stresses. Biological Control 46, 57-71.
Lecam, B., Rouxel, F., Villeneuve, F., 1993. Postharvest pathogens on cold stored carrots - Mycocentrospora-acerina (deighton), the major spoilage fungus. Agronomie 13, 125-133.

Louarn, S., Nawrocki, A., Edelenbos, M., Jensen, D.F., Jensen, O.N., Collinge, D.B., Jensen, B., 2012. The influence of the fungal pathogen Mycocentrospora acerina on the proteome and polyacetylenes and 6-methoxymellein in organic and conventionally cultivated carrots (Daucus carota) during post harvest storage. Journal of Proteomics 75, 962-977.

Magnusson, M.K., Arvola, A., Hursti, U.K.K., Åberg, L., Sjödén, P.O., 2003. Choice of organic foods is related to perceived consequences for human health and to environmentally friendly behaviour. Appetite 40, 109-117.

Mercier, J., Arul, J., Julien, C., 1994. Effect of food preparation on the isocoumarin, 6 -methoxymellein, content of UV-treated carrots. Food Research International 27, 401-404.

Miernyk, J.A., 1997. Molecular analysis of the Arabidopsis cytoplasmic Stress70 chaperone machine. Plant Physiology 114, 1211.

Mooney, B.P., Miernyk, J.A., Greenlief, C.M., Thelen, J.J., 2006. Using quantitative proteomics of Arabidopsis roots and leaves to predict metabolic activity. Physiologia Plantarum 128, 237-250.

Nawrocki, A., Fey, S.J., Goffeau, A., Roepstorff, P., Larsen, P.M., 2001. The effects of transcription regulating genes PDR1, pdr1-3 and PDR3 in pleiotropic drug resistance. Proteomics 1, 1022-1032.

Nawrocki, A., Thorup-Kristensen, K., Jensen, O.N., 2011. Quantitative proteomics by 2DE and MALDI MS/MS uncover the effects of organic and conventional cropping methods on vegetable products. Journal of Proteomics 74, 2810-2825.

Neilson, K.A., Gammulla, C.G., Mirzaei, M., Imin, N., Haynes, P.A., 2010. Proteomic analysis of temperature stress in plants. Proteomics 10, 828-845.

Porras-Alfaro, A., Bayman, P., 2011. Hidden fungi, emergent properties: endophytes and microbiomes. Annual Review of Phytopathology 49, 291-315.

Promputtha, I., Lumyong, S., Dhanasekaran, V., McKenzie, E.H.C., Hyde, K.D., Jeewon, R., 2007. A phylogenetic evaluation of whether endophytes become saprotrophs at host senescence. Microbial Ecology 53, 579-590.

Rahman, L.N., Smith, G.S.T., Bamm, V.V., Voyer-Grant, J.A.M., Moffatt, B.A., Dutcher, J.R., Harauz, G., 2011. Phosphorylation of Thellungiella salsuginea dehydrins tsdhn-1 and tsdhn-2 facilitates cation-induced conformational changes and actin assembly. Biochemistry 50, 9587-9604.

Redman, R.S., Kim, Y.O., Woodward, C.J.D.A., Greer, C., Espino, L., Doty, S.L., Rodriguez, R.J., 2011. Increased fitness of rice plants to abiotic stress via habitat adapted symbiosis: a strategy for mitigating impacts of climate change. PLoS ONE, 6.

Renaut, J., Lutts, S., Hoffmann, L., Hausman, J.F., 2004. Responses of poplar to chilling temperatures: proteomic and physiological aspects. Plant Biology 6, 81-90.

Rosa, L.H., Vieira, M.D.A., Santiago, I.F., Rosa, C.A., 2010. Endophytic fungi community associated with the dicotyledonous plant Colobanthus quitensis (Kunth) Bartl (Caryophyllaceae) in Antarctica. FEMS Microbiology Ecology 73, 178-189.

Schulz, B., Boyle, C., Draeger, S., Römmert, A.K., Krohn, K., 2002. Endophytic fungi: a source of novel biologically active secondary metabolites. Mycological Research 106, 996-1004.

Schwender, J., Goffman, F., Ohlrogge, J.B., Shachar-Hill, Y., 2004. Rubisco without the Calvin cycle improves the carbon efficiency of developing green seeds. Nature 432, 779-782.

Sieber, T., Grünig, C., 2006. Biodiversity of fungal root-endophyte communities and populations, in particular of the dark septate endophyte Phialocephala fortinii s 1. In: Schulz, B.J.E., Boyle, C.J.C., Sieber, T.N. (Eds.), Microbial Root Endophytes. , 9 th ed. Springer, Berlin, Heidelberg, pp. 107-132.

Sundelin, T., Collinge, D., Lübeck, M., 2009. A cultivation independent PCR-based protocol for the direct identification of plant pathogens in infected plant material. European Journal of Plant Pathology 123, 473-476.

Surette, M.A., Sturz, A.V., Lada, R.R., Nowak, J., 2003. Bacterial endophytes in processing carrots Daucus carota L. var. sativus: their localization, population density, biodiversity and their effects on plant growth. Plant Soil 253, 381-390.

Suzuki, Y., Nakabayashi, K., Yoshizawa, R., Mae, T., Makino, A., 2009. Differences in expression of the rbcs multigene family and rubisco protein content in various rice plant tissues at different growth stages. Plant and Cell Physiology 50, 1851-1855.

Thorup-Kristensen, K., Dresbøll, D.B., Kristensen, H.L., 2012. Crop yield, root growth, and nutrient dynamics in a conventional and three organic cropping systems with different levels of external inputs and $\mathrm{N}$ re-cycling through fertility building crops. European Journal of Agronomy 37, 66-82.

Upson, R., Newsham, K.K., Bridge, P.D., Pearce, D.A., Read, D.J., 2009. Taxonomic affinities of dark septate root endophytes of Colobanthus quitensis and Deschampsia antarctica, the two native Antarctic vascular plant species. Fungal Ecology 2, 184-196.

White, T.J., Bruns, T., Lee, S., Taylor, J., 1990. Amplification and direct sequencing of fungal ribosomal RNA genes for phylogenetics. In: Innis, M.A., Gelfand, D.H., Sninsky, J.J., White, T.J. (Eds.), PCR Protocols: a Guide to Methods and Applications. Academic Press, New York, pp. 315-322.

Wilberforce, E.M., Boddy, L., Griffiths, R., Griffith, G.W., 2003. Agricultural management affects communities of culturable root-endophytic fungi in temperate grasslands. Soil Biology and Biochemistry 35, 1143-1154.

Woteki, C.E., Facinoli, S.L., Schor, D., 2001. Keep food safe to eat: healthful food must be safe as well as nutritious. Journal of Nutrition 131, 502S-509S.

Yuan, Z.L., Zhang, C.L., Lin, F.C., 2010. Role of diverse non-systemic fungal endophytes in plant performance and response to stress: progress and approaches. Journal of Plant Growth Regulation 29, 116-126. 